\title{
THE STARBURST IN THE NUCLEUS OF NGC 6764: THE NEAR-IR/RADIO/OPTICAL CONNECTION
}

\author{
MURRAY CAMERON, ANDREAS ECKART, REINHARD GENZEL \\ Max-Planck-Institut für extraterrestrische Physik, Garching, Germany \\ NAOMASA NAKAI \\ Nobeyama Radio Observatory, Nagano, Japan
}

STEFAN WAGNER

Landessternwarte, Heidelberg, Germany

Knowledge of the distribution and excitation of gas close to the nuclei of starburst galaxies is an essential element in the construction of models dealing with intense, but short-lived, star forming events. Building a comprehensive picture of the concentrations of various gaseous components in such regions calls for a multi-wavelength approach: the principal cooling lines of cold $(\mathrm{T} \leq 200 \mathrm{~K})$ molecular gas fall in the millimetre waveband, those of hot $\left(\mathrm{T} \sim 1-3 \times 10^{3} \mathrm{~K}\right)$ excited molecular material in the near-IR, and those pinpointing starburst activity in the optical. In many cases the extents of the emitting regions are on the order of only a few arcseconds.

As part of such a study into the relationship between various near- and circum- nuclear gaseous components in starburst galaxies, we have obtained data on NGC 6764 , a barred spiral classified as a LINER, at resolutions of $1^{\prime \prime}-4^{\prime \prime}$ across the optical, near-infrared and millimetre wavelength regimes. In a previous paper (Eckart et al. 1991) we discussed extensive single dish millimetre and JHK near-IR measurements of the nucleus and disk of NGC 6764 . Here we present new near-IR observations obtained with the MPE FAST instrument (Rotaciuc et al. 1991) at the William Herschel Telescope, along with $2^{\prime \prime}$ resolution ${ }^{12} \mathrm{CO}$ $\mathrm{J}=1-0(115 \mathrm{GHz})$ interferometry measured with the Nobeyama millimeter array and 1 " optical spectroscopy from the $3.5 \mathrm{~m}$ Calar Alto telescope.

Near-IR images, at spatial and spectral resolutions of $\sim 1^{\prime \prime}$ and $300 \mathrm{kms}^{-1}$ respectively, were obtained in the $2.121 \mu \mathrm{m} \mathrm{H}_{2} \mathrm{v}=1-0 \mathrm{~S}(1)$ line, which traces hot molecular material with densities $\sim 5 \times 10^{4} \mathrm{~cm}^{-3}$, excited via the action of shocks or in gas heated by intense UV or X-ray fluxes (see van der Werf et al., these proceedings). These data are presented in the accompanying figure together with our Nobeyama $\mathrm{CO}$ measurements and a $5 \mathrm{GHz}$ VLA radio continuum map (Wilson \& Willis 1980). These data indicate that the excited $\mathrm{H}_{2}$ gas is extended on scales of a few arcseconds and that the emission shows prominent extensions to the south and south-east, similar to those seen in the radio continuum map. Although the ${ }^{12} \mathrm{CO}$ map, which traces molecular gas with temperatures of 10 $20 \mathrm{~K}$ and number densities of a few times $10^{4} \mathrm{~cm}^{-3}$, confirms that a large fraction of the emission arises from the inner $5^{\prime \prime}$, it also reveals components which are 
associated with the spiral arms close to the nucleus.

Our optical spectrum of the nuclear region of this galaxy (not shown here) reveals a prominent $4665 \AA$ feature comprising emission in the lines of NIII, CIII/CIV and HeII which is only seen in a small number of galaxies and has been attributed to the presence of Wolf-Rayet stars. Since these are highly evolved descendants of the most massive $O$ stars (with initial masses $\geq 35 \mathrm{M}_{\odot}$ ), the presence of this feature is regarded as direct evidence for recent massive star formation. Deconvolution of the width of this feature with the instrument point spread function indicates that the emitting region has a FWHM of $\sim 1.9^{\prime \prime}$, corresponding to $\sim 200 \mathrm{pc}$. However, $\sim 30 \%$ of the emission arises from relatively low surface brightness regions which extend over $\sim 4^{\prime \prime}$. As such, it appears that massive star formation is taking place throughout the region where the hot molecular gas is located.

In summary, the radio, near-infrared and optical data indicate that a recentlytriggered massive starburst is taking place in the central 200pc of NGC 6764 . The mass of hot $\mathrm{H}_{2}$ is $\sim 1-2 \times 10^{3} \mathrm{M}_{\odot}$, while the cold nuclear molecular mass is $\sim 2-3 \times 10^{8} \mathrm{M}_{\odot}$. A full account of this work, including analysis of a range of nearIR lines $(2.17 \mu \mathrm{m} \mathrm{Br} \gamma, 1.64 \mu \mathrm{m}$ [FeII], 2.06 $\mu \mathrm{m} \mathrm{HeI})$, will be presented elsewhere.
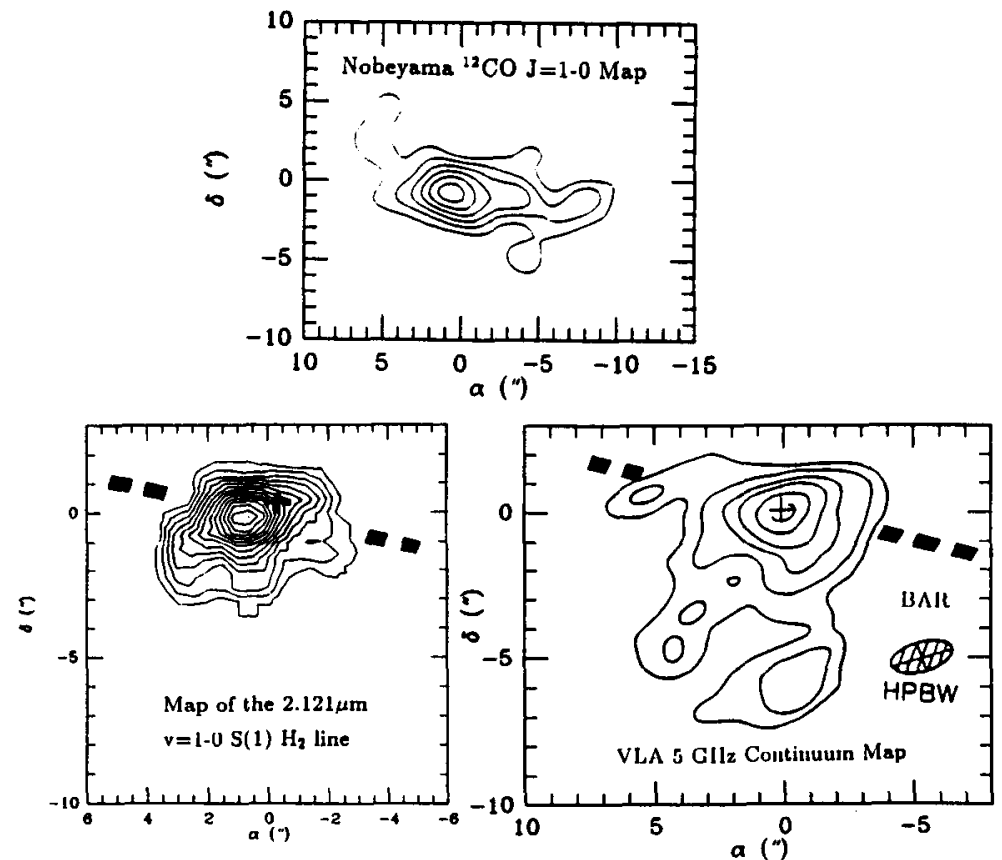

REFERENCES

Eckart A., Cameron M., Jackson J.M., Genzel R., Harris A.I., Wild W. \& Zinnecker H., 1991, Ap.J., 372, 67.

Rotaciuc V., Krabbe A., Cameron M., Drapatz S., Genzel R., Sternberg A. \& Storey J.W.V., 1991, Ap.J., 370, L23.

Wilson A.S. \& Willis A.G., 1980, Ap.J., 240, 429. 